Gut, 1974, 15, 630-635

\title{
Occurrence of cholesterol crystals in human bile
}

\author{
WILLEM VAN DER LINDEN AND FUMIO NAKAYAMA \\ From the Surgical Department, Centrallasarettet, Östersund, Sweden and the Surgical Clinic, Kyushu \\ University Faculties of Medicine and of Health Science, Fukuoka, Japan
}

SUMMARY The occurrence of cholesterol crystals was studied in 20 consecutive gallstone patients with functioning gallbladders. The frequency with which crystals were found rose sharply with the number of stones. Gallbladder bile was found more often to contain cholesterol crystals than hepatic bile of the same individual. Such crystals were absent in $T$ tube drain bile from 10 consecutive choledochostomy patients, studied after the reestablishment of the enterohepatic circulation for at least five days. In gallstone patients in whom the gallbladder was visualized at cholecystography the hepatic bile contained cholesterol crystals more often than in patients with gallbladders not so visualized. In the latter patients the crystals tended to disappear after prolonged fasting. Bile analysis showed hepatic bile of patients with non-functioning gallbladders to be less lithogenic than bile in cases with functioning gallbladders. In the former group bile contained relatively more chenodeoxycholic acid than in the latter. The composition of bile with cholesterol crystals did not differ significantly from that of bile without crystals. In the final analysis it is important to identify possible factors responsible for the precipitation of cholesterol from supersaturated bile.

Although a vast body of information on gallstone formation has assiduously been accumulated during the last few decades, very little is known about the conditions under which cholesterol crystals are found in bile. This may seem amazing as the cholesterol crystal is the obvious precursor of the cholesterol stone. One reason may be that so much work has been done by chemists compared with that by surgeons and other clinicians. When bile samples are taken from the patient to the investigating chemist the often inevitable loss of time with cooling or freezing of the samples makes a later search for crystals less meaningful. Rains (1964) has shown that changes in temperature are highly important for the cholesterol-holding capacity of bile salt solutions. Another reason perhaps is that a study of the occurrence of cholesterol crystals is likely to start with a comparison of gallbladder bile from gallstone patients with that from patients without such stones. Such a comparison will reveal the frequent occurrence of crystals in the former and their virtual absence in the latter group. This banal finding will doubtless be interpreted as showing that where there are stones there are also crystals, a disencouraging conclusion which hardly raises hopes of

Please address requests for reprints to Dr W. van der Linden, Centrallasarettet, S831 01 Östersund, Sweden.

Received for publication 28 March 1974. sensational revelations on further study. Still, as the microscopic examination of freshly sampled bile is a very simple procedure we decided to embark on such a study. The results are reported here.

\section{Material and Methods}

OCCURRENCE OF CHOLESTEROL CRYSTALS IN GALLBLADDER AND HEPATIC BILE

Twenty consecutive patients in whom cholecystography had shown gallstones in well functioning gallbladders were studied. At operation a needle was inserted into the fundus of the gallbladder and the bile aspirated. After identification of the cystic duct the common duct was cannulated via the cystic duct and a sample of hepatic bile was collected. The samples were thoroughly mixed and $1 \mathrm{ml}$ of each was taken for immediate investigation. After centrifugation the sediment was examined microscopically in direct and polarized light and the presence or absence of cholesterol crystals was noted. Two independent assessors rated the occurrence of crystals in each sample according to a four-grade scale. According to the same scale a comparison was made between the hepatic and the gallbladder bile sample of each individual patient. After the operation was completed the number of 
stones in the gallbladder was counted and the cases were divided into three groups: (1) those with solitary stones, (2) those with two-five stones, and (3) those with multiple stones, ie, six or more.

\section{OCCURRENCE OF CHOLESTEROL CRYSTALS}

IN T-TUBE BILE

Ten consecutive patients in whom the common duct had been explored and T-tube drainage had been performed in the course of gallstone surgery, were studied. The choledochostomy tube was closed for at least five days in order to re-establish the enterohepatic circulation after which a bile sample was taken in the morning after an overnight fast. These samples were studied as described above.

\section{FASTING AND OCCURRENCE OF CHOLESTEROL CRYSTALS}

Twenty consecutive patients in whom the gallbladder was not visualized during cholecystography were randomly divided into two equal groups. One group was operated upon early in the morning, the patients having fasted for nine or 10 hours. The other group was operated upon late in the afternoon. In these latter patients fasting had lasted for at least 15 hours. During this fast no intravenous fluid other than saline was given. At operation hepatic bile was collected and immediately investigated as described above. In all these patients gallstones were found at operation.

Twenty consecutive patients in whom cholecystography had shown gallstones in well functioning gallbladders were also randomly divided into two groups which were treated in the same manner. One group was operated after an overnight fast and one group after a prolonged fast. At operation hepatic bile was sampled and investigated.

\section{Bile Analysis}

Bile analysis was performed in all patients except two in whom the volume of bile was not sufficient. It consisted of preliminary solvent partition followed by gas liquid chromotography as described earlier (Nakayama, 1967). Bilirubin was determined with the diazo reaction according to the method described by Malloy and Evelyn (1937). Bile was extracted with 20 times its volume of ethanol and the extract was used for the following determinations: cholic acid was determined with the Pettenkofer reaction according to Irvin, Johnston, and Kopala (1944), phosphorus by Bartlett's (1959) method, and cholesterol with the Liebermann-Burchard reaction as described by Sperry (1950) after partitioning between $70 \%$ cholesterol and petroleum ether. In order to prepare the bile acid sample for gas liquid chromotography, part of the $70 \%$ ethanol extract containing bile acid was evaporated to dryness and subjected to alkaline hydrolysis. Free bile acid was extracted with ether and subjected to gas liquid chromatography on a $3.8 \%$ silicone gum rubber UCCW-982 on 80-100 mesh high performance Chromosorb $\mathrm{W}$ at $230^{\circ}$ after methylation and acetylation with acetic anhydride and pyridine at $115^{\circ}$ for four hours (Plattner and Heusser, 1944). For further details of the methods used, together with particulars concerning accuracy and recovery studies, the reader is referred to an earlier communication (Nakayama, 1967).

Phospholipids were calculated by multiplying the phosphorus value by the factor 25 , and, since biliary phospholipids are composed mainly of lecithin, this was taken as an estimate of the lecithin value. The percentages of the total moles of the three main constituents, ie, cholesterol, bile acids, and lecithin, were then calculated and plotted on triangular coordinates as suggested by Admirand and Small (1968).

\section{Results}

Table I shows the occurrence of cholesterol crystals as related to the number of stones in the gallbladder. As seen in table I the frequency with which crystals are found rises sharply with the number of stones. This holds true both for hepatic bile and for gallbladder bile. The number of cases in each single cell in these two columns is very small. It is therefore perhaps easiest to compare cases with multiple stones, ie, six stones or more, with all other cases as to the occurrence of crystals, regardless of their number. Doing so we find crystals present in the hepatic bile of 10 out of 11 cases with multiple stones as compared with one out of nine cases with solitary or a few stones. With Fisher's test (Siegel, 1956) this difference is statistically significant $(P<0.01)$. In gallbladder bile crystals were found in all the 11 multiple stone cases without exception but they were absent in five out of the nine other

\begin{tabular}{|c|c|c|c|c|c|c|c|c|}
\hline \multirow[t]{3}{*}{ No. of Stones } & \multicolumn{8}{|c|}{ Crystals } \\
\hline & \multicolumn{4}{|c|}{ Hepatic Bile } & \multicolumn{4}{|c|}{ Gallbladder Bile } \\
\hline & - & + & ++ & $++t^{1}$ & - & + & ++ & +++ \\
\hline $\begin{array}{r}1 \\
2-5 \\
\geqq 6\end{array}$ & $\begin{array}{l}6 \\
2 \\
1\end{array}$ & $\begin{array}{l}0 \\
1 \\
7\end{array}$ & $\begin{array}{l}0 \\
0 \\
2\end{array}$ & $\begin{array}{l}0 \\
0 \\
1\end{array}$ & $\begin{array}{l}3 \\
2 \\
0\end{array}$ & $\begin{array}{l}1 \\
1 \\
1\end{array}$ & $\begin{array}{l}2 \\
0 \\
4\end{array}$ & $\begin{array}{l}0 \\
0 \\
6\end{array}$ \\
\hline
\end{tabular}

Table I Occurrence of cholesterol crystals related to number of stones in the gallbladder

- denotes no crystals, + sporadic or few, ++ many, and +++ many and large crystals 
cases. With the same test this difference is again statistically significant $(P<0.05)$. Table I shows further that in the majority of the multiple stone cases the gallbladder bile crystals were rated as many and large. This classification was not used in any case with one or a few stones.

Figure 1 shows the occurrence of crystals in the hepatic and the gallbladder bile sample of each individual patient. As seen in fig 1 crystals were absent in both hepatic and gallbladder bile of five patients. In all remaining cases except two, the occurrence of crystals in the gallbladder bile was rated higher than in the hepatic bile. With the sign test (Siegel, 1956) this difference between the two series of samples is statistically highly significant $(\mathrm{P}<0.001)$.

In none of the $10 \mathrm{~T}$-tube patients were crystals found in the hepatic bile collected after cholecystectomy and the reestablishment of the enterohepatic circulation. During operation crystals had been present in the hepatic bile of five of them.

\section{Hepatic}

Gallbladder

\section{Crystals:}

many and large

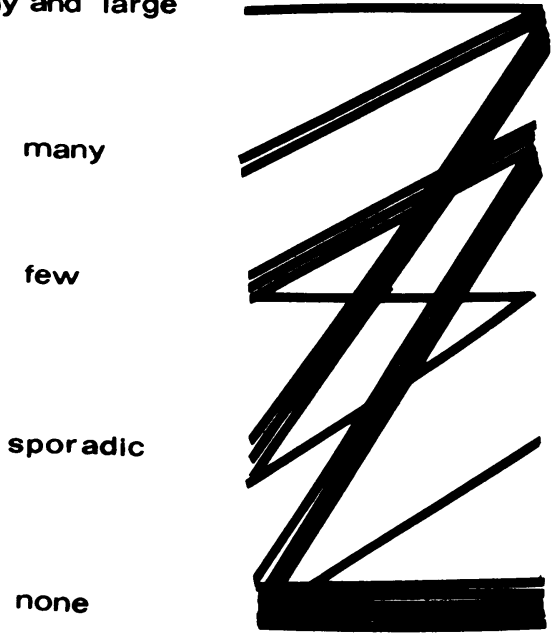

Fig 1 Cholesterol crystals in hepatic and gallbladder bile of each individual patient
As seen in table II there was a significant difference in the occurrence of crystals in hepatic bile of patients with visualized as compared with those with non-visualized gallbladders $(P=0.026$ according to Mainland, Herrera, and Sutcliffe, 1956). In the former group crystals were found more often than in the latter. Table II shows that this difference is caused by the total absence of crystals in patients with non-functioning gallbladders subjected to prolonged fasting. These cases differ significantly from similar patients who had fasted less than 12 hours $(P=0.011)$. Such a difference was not found in the group in which the gallbladder was visualized at cholecystography. In this latter group prolonged fasting did not have any influence on the occurrence of crystals in hepatic bile. Figures 2 and 3 show the composition of the hepatic bile plotted on triangular coordinates. In the group with functioning gallbladders nine out of 18 samples were situated inside the micellar zone as compared with 16 out of 20 cases with non-functioning gallbladders. This difference between the two groups is not statistically significant, When, however, the lithogenic index, described by Metzger, Heymsfield, and Grundy (1972), is calculated for each sample the difference between the groups is significant at the

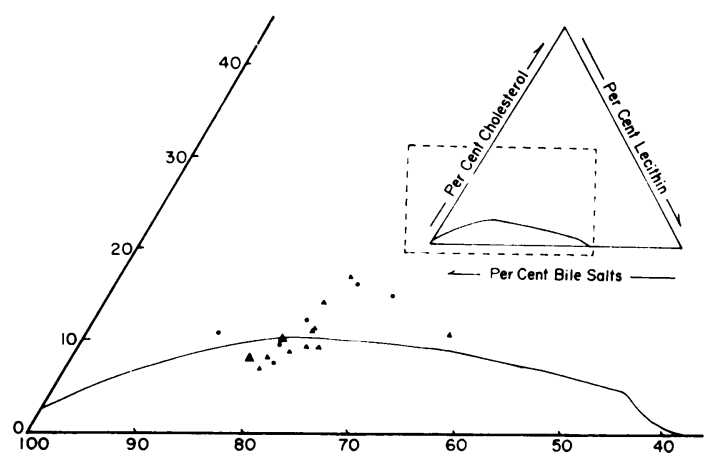

Fig 2 Composition of hepatic bile in group with functioning gallbladder. Triangular phase diagram according to Admirand and Small (1968): with cholesterol crystals $\Delta$, with abundant cholesterol crystals $\boldsymbol{\Delta}$, without cholesterol crystals $\bullet$

\begin{tabular}{|c|c|c|c|c|c|c|}
\hline \multirow{2}{*}{$\begin{array}{l}\text { Crystals in } \\
\text { Hepatic Bile }\end{array}$} & \multicolumn{3}{|c|}{ Non-visualized Gallbladder } & \multicolumn{3}{|c|}{ Visualized Gallbladder } \\
\hline & $\begin{array}{l}\text { Fasting } \\
<12 \mathrm{hr}\end{array}$ & $\begin{array}{l}\text { Fasting } \\
>12 \mathrm{hr}\end{array}$ & $\Sigma$ & $\begin{array}{l}\text { Fasting } \\
<12 \mathrm{hr}\end{array}$ & $\begin{array}{l}\text { Fasting } \\
>12 \mathrm{hr}\end{array}$ & $\Sigma$ \\
\hline \multirow[t]{2}{*}{$\begin{array}{l}\text { Present } \\
\text { Absent }\end{array}$} & $\begin{array}{l}6 \\
4\end{array}$ & $\begin{array}{r}0 \\
10\end{array}$ & $\begin{array}{r}6 \\
14\end{array}$ & $\begin{array}{l}7 \\
3\end{array}$ & $\begin{array}{l}7 \\
3\end{array}$ & $\begin{array}{r}14 \\
6\end{array}$ \\
\hline & 10 & 10 & 20 & 10 & 10 & 20 \\
\hline
\end{tabular}

Table II Crystals in hepatic bile in visualized and non-visualized gallbladders in fasting more and less than 12 hours 


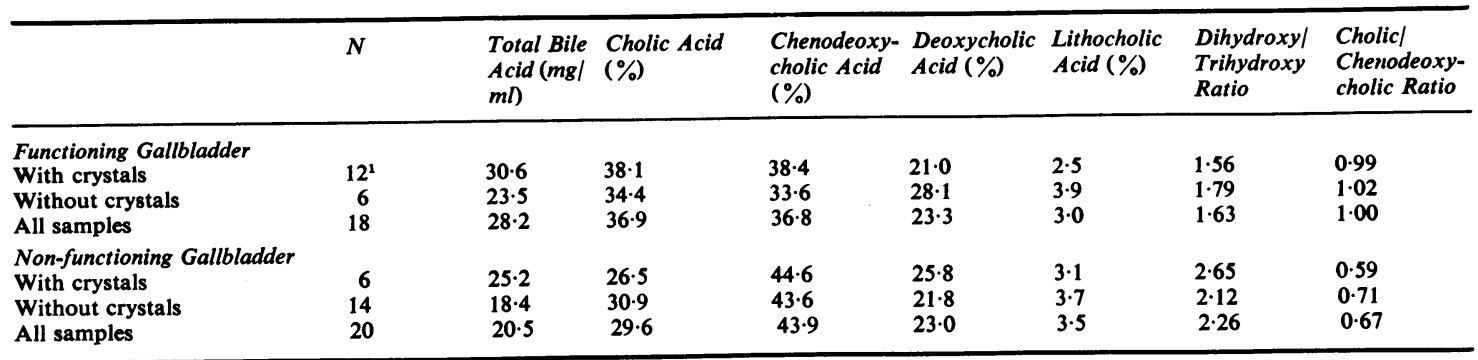

Table III Bile acid pattern in cases with functioning and non-functioning gallbladders (hepatic bile)

${ }^{1}$ Two samples in this group were too small for analysis.

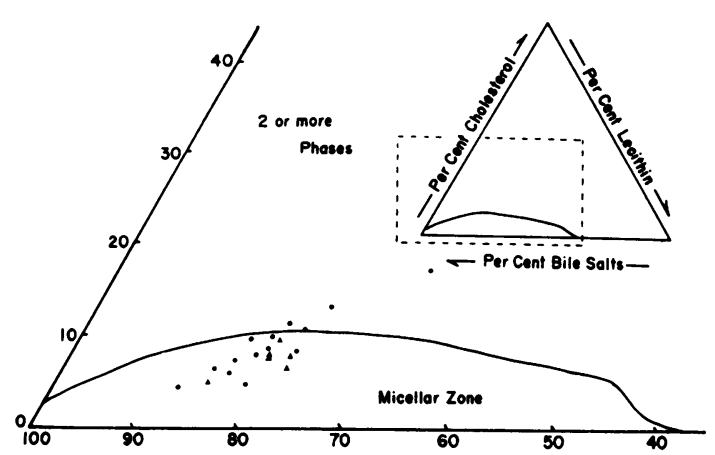

Fig 3 Composition of hepatic bile in group with nonfunctioning gallbladder. Triangular phase diagram according to Admirand and Small (1968): with cholesterol crystals $\triangle$, without cholesterol crystals

$2 \%$ level, when subjected to the Mann-Whitney $U$ test (Siegel, 1956). Furthermore, it is apparent from figs 2 and 3 that there is no systematic difference between samples with and without cholesterol crystals. Neither was there a clear difference between patients with non-functioning gallbladders operated after prolonged fasting and those operated earlier.

Finally table III gives the bile acid composition in the two groups. As seen in this table there are no systematic differences between samples with and those without crystals. On the other hand there are significant differences between cases with functioning and those with non-functioning gallbladders. In the latter group there is relatively more chenodeoxycholic acid. This results in a higher dihydroxy/ trihydroxy bile acid ratio and a lower cholic/chenodeoxycholic ratio in this group. When subjected to the Mann-Whitney U test this group difference is statistically significant.

\section{Discussion}

In the first part of our study we assessed the occur- rence of cholesterol crystals in gallbladder and hepatic bile. The bile samples were obtained from well functioning gallbladders containing different numbers of stones. The results (see table I) clearly show that with more stones in the gallbladder there are also more and larger cholesterol crystals. This applies both to gallbladder and to hepatic bile. A plausible explanation for this finding is supplied by the study of Wolpers (1972). Using electron microscopy, Wolpers (1972) found the size of the cholesterol crystals to be very different in different stones. In multiple cholesterol stones there were fine slices of crystals with a large surface as compared with large plates with a small surface in solitary stones. As seen in table I, with these latter stones in the gallbladder we often failed to find any crystals at all. Wolpers (1972) found such stones to be less liable to dissolution. It seems reasonable to assume that from concrements so built crystals loosen less easily.

There is another finding which warrants comment, namely, the finding that the occurrence of crystals in hepatic bile is related to the number of stones in the gallbladder. Clearly, a direct or indirect influence of the number of stones on the composition of liver bile is difficult to imagine. The obvious explanation, therefore, is that in cases with a functioning gallbladder, bile found in the hepatic duct at operation is not true hepatic, ie, liver, bile but a mixture of such bile with gallbladder bile.

We continued our study by comparing in each individual patient the occurrence of cholesterol crystals in hepatic bile and in gallbladder bile. As seen in fig 1 a significant difference was ascertained. Gallbladder bile contained often more but never fewer crystals than did hepatic bile. At first sight this may seem a banal finding as bile is concentrated in the gallbladder. It should be remembered, however, that under fasting conditions hepatic bile has been shown to be consistently more lithogenic than gallbladder bile (Metzger, Adler, Heymsfield, and Grundy, 1973). 
Assuming that the bile found at operation in the common duct is a mixture of liver bile and gallbladder bile, the question now arises whether true hepatic bile ever contains cholesterol crystals at all. As seen in table II, in cases with gallbladders not being visualized at cholecystography, cholesterol crystals were found significantly less frequently than in cases which were capable of being visualized. But, although less frequently, in a number of nonvisualizing cases crystals did occur in the hepatic duct. This, however, is no definite proof, for even if a gallbladder cannot be visualized at cholecystography it may function just enough to contaminate the hepatic bile with some gallbladder bile. Still, in the course of our study, we met with cases in which such contamination could definitely be excluded. These were cases in which the cystic duct was occluded and in which the bladder contained 'white bile.' In spite of this proven occlusion of the cystic duct, some of these cases had cholesterol crystals in the hepatic bile. Clearly therefore true hepatic bile may contain cholesterol crystals.

Hepatic bile sampled after cholecystectomy and the reestablishment of the enterohepatic circulation did, on the other hand, never contain cholesterol crystals. This is in accordance with the concept that after cholecystectomy hepatic bile is stabilized as regards cholesterol solubilization. Such a normalization of the hepatic bile has been shown in a number of studies (Simmons, Ross, and Bouchier, 1972; Shaffer, Braasch, and Small, 1972; Nakayama and van der Linden, 1974). The finding contradicts the only study claiming that following cholecystectomy bile still remains saturated as regards cholesterol (Bell, Almond, Vlahcevic, Gregory, and Swell, 1973) and it stresses the essential role the gallbladder seems to play in the production of lithogenic hepatic bile.

Analysis of the bile samples revealed that hepatic bile of patients with gallstones may be situated well inside the micellar zone as defined by Admirand and Small (1968). However, when this zone is defined according to the calculations of Holzbach, Marsh, Olszewski, and Holan (1973) all samples were situated outside that zone. Although we do not want to enter into any controversy regarding the exact boundaries of the zone, this finding may be taken as supporting the calculations of these latter authors whose findings are in agreement with those of Hegardt and Dam (1971).

Metzger, Heymsfield, and Grundy (1972) have proposed a lithogenic index which, because of its numerical nature, is very suitable for the statistical comparison of groups of samples. Using this index we found hepatic bile of patients with non-functioning gallbladders to be less lithogenic than that of patients in whom the gallbladder was visualized at cholecystography. This is in accordance with recent findings of Cahlin, Jönsson, Nilsson, and Scherstén (1973). Admittedly the difference between groups so compared is not very pronounced and there is a considerable overlap. Still this group difference tends to give further support to the rôle of the gallbladder in gallstone formation.

Comparison of samples in which cholesterol crystals were present with samples without such crystals failed to reveal a systematic difference. This somewhat surprising finding is in agreement with the study of Holzbach, Marsh, Olszewski, and Holan (1973) and in contrast to the report of Admirand and Small (1968).

In patients with non-functioning gallbladders prolonged fasting resulted in the disappearance of cholesterol crystals from hepatic bile. The chemical composition did not, on the other hand, seem to alter significantly. It should be pointed out, however, that the subgroups studied are small and further study is no doubt warranted. Fasting hepatic bile has been convincingly demonstrated to be consistently more lithogenic than gallbladder bile or hepatic bile obtained after feeding (Metzger, Adler, Heymsfield, and Grundy, 1973). This effect of fasting has also been demonstrated in other primates (McSherry, Glenn, and Javitt, 1971) and is most probably due to the sequestration of bile acids which takes place in the gallbladder during fasting. In other words, if the gallbladder is intact there is during fasting a physiological interruption of the enterohepatic circulation of bile acids. Our finding that in patients with functioning gallbladders cholesterol crystals were just as frequent after prolonged fasting as after fasting for less than 12 hours, while there was no clear difference in bile composition, is not in disagreement with this hypothesis. Probably a well functioning gallbladder can interrupt the enterohepatic circulation for quite a long time. When fasting is prolonged the hepatic bile is and remains lithogenic.

On the other hand, an interruption of the enterohepatic circulation of bile can, clearly, not take place if the function of the gallbladder is eliminated by a pathological process, ie, an inflammation or an occlusion of the cystic duct by a stone. In such cases there is a more or less continuous secretion of bile which is nearly constantly dripping into the duodenum. Under these circumstances the effect of prolonged fasting may make itself felt in a very different way. Whether there is a change in bile composition under these circumstances cannot be excluded because of the relative smallness of the subgroups studied, but it was clearly not apparent 
in the present study. In spite of this cholesterol crystals tended to disappear.

The difference in bile acid pattern between cases with functioning and those with non-functioning gallbladders-more chenodeoxycholic acid in the latter group-is not easily explicable. It is known that under conditions of increased loss of bile acids there is a shift towards cholic acid (van der Linden and Nakayama, 1969). With the continuous secretion of bile which ensues after elimination of the gallbladder, the recirculation of bile acids may become more complete. Under such conditions of presumably decreased loss of bile acids there is perhaps a shift in the opposite direction, ie, towards chenodeoxycholic acid.

During the last decade gallstone research has been wholly concentrated on identifying factors responsible for the supersaturation of bile with cholesterol. Although this no doubt is a crucial issue we are more and more forced to admit that supersaturation alone cannot explain when gallstones do and when they do not appear. For instance, in the hamster model treatment with a cholesterol biosynthesis inhibitor (van der Linden, Nakayama, and Bergman, 1971) and with hyodeoxycholic acid (Wheeler, 1973) gave paradoxical results: highly lithogenic bile but prevention of gallstone formation. Now it seems that in human bile something similar may be found. In supersaturated bile cholesterol crystals are present or absent without any clear relationship to bile composition. Still their appearance and disappearance seems to be determined by certain factors. Further identification of these factors could prove to be of the utmost importance.

\section{References}

Admirand, W. H., and Small, D. M. (1968). The physicochemical basis of cholesterol gallstone formation in man. J. clin. Invest., 47, 1043.

Bartlett, G. R. (1959). Phosphorus assay in column chromatography. J. biol. Chem., 234, 466-468.

Bell, C. C. J., Almond, H. R., Vlahcevic, Z. R., Gregory, D. H., and Swell, C. (1973). The effect of cholecystectomy on bile acid pool size, kinetics and biliary lipid composition in patients with cholesterol gallstones. (Abstr.) Gastroenterology, 64, 193/879.

Cahlin, E., Jönsson, J., Nilsson, J., and Scherstén, T. (1973). Biliary lipid composition in normolipidemic and prebeta hyperlipoproteinemic gallstone patients. Influence of sucrose feeding of the patients on the biliary lipid composition. Scand. J. Gastroent., 8, 449-456.

Hegardt, F. G., and Dam, H. (1971). The solubility of cholesterol in aqueous solutions of bile and lecithin. Z. Ernährungsw., 10, 223-233.

Holzbach, R. T., Marsh, M., Olszewski, M., and Holan, K. (1973). Cholesterol solubility in bile: evidence that supersaturated bile is frequent in healthy man. J. clin. Invest., 52, 1467-1479.

Irvin, J. L., Johnston, C. G., and Kopala, J. (1944). A photometric method for the determination of cholates in bile and blood. J. biol. Chem., 153, 439-457.

McSherry, C. K., Glenn, F., and Javitt, N. B. (1971). Composition of basal and stimulated hepatic bile in baboons and the formation of cholesterol gallstones. Proc. nat, Acad. Sci. (Wash.), 68, 1564-1568.

Mainland, D., Herrera, L., and Sutcliffe, M. I. (1956). Statistical Tables for Use with Binomial Samples. University College of Medicine, New York.

Malloy, H. T., and Evelyn, K. A. (1937). The determination of bilirubin with the photo-electric colorimeter. J. biol. Chem., $119,481-490$

Metzger, A. L., Adler, R., Heymsfield, S., and Grundy, S. M. (1973). Diurnal variation in biliary lipid composition. New Engl. J. Med., 288, 333-336.

Metzger, A. L., Heymsfield, S., and Grundy, S. M. (1972). The lithogenic index $-a$ numerical expression for the relative lithogenicity of bile. Gastroenterology, 62, 499-501.

Nakayama, F. (1967). Quantitative microanalysis of bile. J. Lab. clin. Med., 69, 594-609.

Nakayama, F., and van der Linden, W. (1970). Bile from gallbladder harbouring gallstone: can it indicate stone formation? Acta chir. scand., 136, 605-610.

Nakayama, F., and van der Linden, W. (1974). Role of gallbladder in gallstone formation Acta chir. scand., 140, 45-49.

Plattner, P. A., and Heusser, H. (1944). Úber Steroide und Sexualhormone. Uber Beziehung zwischen Konstitution und optischer Drehung in der Cholsäure-Reihe. Helv. chim. acta, 27, 748

Rains, A. J. H. (1964). Gallstones. Causes and Treatment. Heinemann, London.

Shaffer, E. A., Braasch, J. W., and Small, D. M. (1972). Bile composition at and after surgery in normal persons and patients with gallstones. New Engl. J. Med., 287, 1317-1322.

Siegel, S. (1956). Non-Parametric Statistics for the Behavioral Sciences. MacGraw-Hill, New York.

Simmons, F., Ross, A. P. J., and Bouchier, I. A. D. (1972). Alterations in hepatic bile composition after cholecystectomy. Gastroenterology, 63, 466-471.

Sperry, W. M., and Webb, M. (1950). A revision of the SchoenheimerSperry method for cholesterol determination. J. biol. Chem., 187, 97-106.

van der Linden, W., and Nakayama, F. (1969). Change of bile composition in man after administration of cholestyramine (a gallstone dissolving agent in hamsters). Acta chir. scand., $135,433-438$.

van der Linden, W., Nakayama, F., and Bergman, F. (1971). Effect of cholesterol biosynthesis inhibitor AY-9944 on gallstone formation in hamsters. Acta chir. scand., 137, 355-360.

Wheeler, H. O. (1973). Biliary excretion of bile acids, lecithin and cholesterol in hamsters with gallstones. Gastroenterology, $65,92-103$

Wolpers, C. (1972). Relapses after spontaneous dissolution of gallstones. In Bile Acids in Human Diseases, edited by $P$. Back and W. Gerok. Schattauer, Stuttgart. 